\title{
RT States: systematic annotation of the human genome using cell type-specific replication timing programs
}

\author{
Axel Poulet ${ }^{1}$, Ben Li ${ }^{1}$, Tristan Dubos ${ }^{2}$, Juan Carlos Rivera-Mulia ${ }^{3, \dagger}$, \\ David M. Gilbert ${ }^{3}$ and Zhaohui S. Qin ${ }^{1, *}$
}

${ }^{1}$ Department of Biostatistics and Bioinformatics, Rollins School of Public Health, Emory University, Atlanta, GA 30322, USA, ${ }^{2}$ Laboratoire de Génétique, CHRU-Nancy, 54000 Nancy, France and ${ }^{3}$ Department of Biological Science, Center for Genomics and Personalized Medicine, Florida State University, Tallahassee, FL 32304, USA

*To whom correspondence should be addressed.

${ }^{\dagger}$ Present address: Department of Biochemistry, Molecular Biology, and Biophysics, University of Minnesota Medical School, Minneapolis, MN 55455, USA.

Associate Editor: John Hancock

Received on August 29, 2018; revised on November 5, 2018; editorial decision on November 19, 2018; accepted on November 21, 2018

\begin{abstract}
Motivation: The replication timing (RT) program has been linked to many key biological processes including cell fate commitment, 3D chromatin organization and transcription regulation. Significant technology progress now allows to characterize the RT program in the entire human genome in a highthroughput and high-resolution fashion. These experiments suggest that RT changes dynamically during development in coordination with gene activity. Since RT is such a fundamental biological process, we believe that an effective quantitative profile of the local RT program from a diverse set of cell types in various developmental stages and lineages can provide crucial biological insights for a genomic locus.

Results: In this study, we explored recurrent and spatially coherent combinatorial profiles from 42 RT programs collected from multiple lineages at diverse differentiation states. We found that a Hidden Markov Model with 15 hidden states provide a good model to describe these genome-wide RT profiling data. Each of the hidden state represents a unique combination of RT profiles across different cell types which we refer to as 'RT states'. To understand the biological properties of these RT states, we inspected their relationship with chromatin states, gene expression, functional annotation and 3D chromosomal organization. We found that the newly defined RT states possess interesting genome-wide functional properties that add complementary information to the existing annotation of the human genome.

Availability and implementation: $\mathrm{R}$ scripts for inferring HMM models and Perl scripts for further analysis are available https://github.com/PouletAxel/script_HMM_Replication_timing.
\end{abstract}

Contact: zhaohui.qin@emory.edu

Supplementary information: Supplementary data are available at Bioinformatics online.

\section{Introduction}

The functional significance of genome organization at the sequence level of many regions in the human genome remains poorly understood despite the completion of the human genome sequencing project more than 15 years ago. Large international consortia such as
ENCODE (Dunham et al., 2012), REMC (Bernstein et al., 2010) and IHEC (Bae, 2013) have demonstrated that there are many functional elements present throughout the genome that play interesting and important roles in structuring the human genome. The discovery of these functional elements highlights the importance of 
defining, cataloging and characterizing the diverse regions that remain poorly understood. It is important to advance the field through a variety of methods, integrating different experimental designs and findings, allowing the definition of structure like topologically associating domain (TADs) (Dixon et al., 2012; Hou et al., 2012; Sexton et al., 2012) or Lamin Associated Domains (LADs) (Guelen et al., 2008; Meuleman et al., 2013). Alternatively, statistical modeling applied on these experimental data created a new, composite types of genomic elements. As a prime example, Ernst and Kellis exploited ChIP-seq data of histone modifications to classify the genome into chromatin states using the Hidden Markov Model (HMM) (Ernst and Kellis, 2010, 2012; Ernst et al., 2011). They found that many of these chromatin states are correlated with particular cis-regulatory elements, such as enhancers or insulators. The appealing feature of the chromatin state concept is its ability to crystallize complex and biologically meaningful patterns into a few numbers using a large collection of epigenomic profiles that contain lots of variation, overlap and redundancy. We believe the same strategy can be applied to other types of data. In this study, we annotate the genome using a rich set of genome-wide replication timing (RT) programs derived from multiple human cell types (Hiratani et al., 2008; Ryba et al., 2011a). The RT programs represent the genome duplication occurring in a defined temporal order in human cells.

In humans, the RT program is dynamic and cell type-specific. The difference between types of differentiated cells, and even stem cells and their progenitors can be dramatic. For example, half of the genome undergoes RT changes during cell differentiation of embryonic stem cells (Hiratani et al., 2009; Pope and Gilbert, 2013). These dynamic changes in RT occur in units of 400-800 kb known as replication domains (RDs) (Hansen et al., 2010; Hiratani et al., 2008, 2010). RT alterations have also been linked to many diseases (Gerhardt et al., 2014; Rivera-Mulia et al., 2017; Ryba et al., 2012; Sasaki et al., 2017). Recent studies have revealed an association between RT and higher-order chromosomal structure (TADs and subnuclear compartment A and B) (Hiratani et al., 2010; Hu et al., 2013b; Liu et al., 2016; Pope et al., 2014; Rivera-Mulia et al., 2015), as well as gene expression regulation (Rivera-Mulia et al., 2015), supporting the hypothesis that the genome is partitioned into smaller replication units of replication (Rivera-Mulia and Gilbert, 2016). In general, early replication is correlated with elevated transcriptional activity (Hatton et al., 1988; Hiratani et al., 2010). Researchers have classified RDs as constitutive or developmentally regulated, depending on how consistent the replication occurs across different cell types (Hatton et al., 1988; Hiratani et al., 2010). RD boundaries are stable across different cell types, but the changes of $\mathrm{RT}$ on the developmental domains are correlated with changes in sub-nuclear position, chromatin structure and transcriptional state.

The introduction of RDs helps put context to a genomic locus from the RT perspective, but the concept is qualitative. As more RT profiles of more cell types becomes available, we gain the opportunity to characterize RT dynamics in a more refined and quantitative fashion. In this study, we use $42 \mathrm{RT}$ profiles collected from 25 cell types and differentiation intermediates (Rivera-Mulia et al., 2015), and apply a HMM based approach to break down the genome into 15 RT states. The goals are 2-fold: first, identify frequent combinatorial RT patterns among a compendium of cell types; second, annotate the genome by assigning an RT state to every locus. Note that unlike chromatin states (Ernst and Kellis, 2010, 2012; Ernst et al., 2011), RT states defined here remain unchanged across cell types since they represent the behavior of chromatin across a variety of cell types, rather than the composition of chromatin in a particular cell type. The 25 cell types and the differentiation intermediates have been strategically selected to represent the diversity among all the cell types in human and represent developmental stages of the three germ layers (Rivera-Mulia et al., 2015). Finally, the RT states described here represent a more accurate and quantitative means of defining constitutive early, constitutive late or developmental RDs, which adds a novel quantitative annotation of the functional properties of the human genome.

\section{Materials and methods}

\subsection{RT data}

We analyzed 42 genome-wide RT datasets of Repli-chip previously published (Rivera-Mulia et al., 2015), representing 25 cell types and differentiation intermediates of human development. The genome was first divided into bins of $50 \mathrm{~kb}$, then RT value was calculated for each bin. The 42 datasets include human embryonic stem cells (hESC), primary cells and established cell lines representing intermediate stages of endoderm, mesoderm, ectoderm and neural crest development (Supplementary Table S1) (Hiratani et al., 2010; Pope et al., 2011; Rivera-Mulia et al., 2015; Ryba et al., 2011b; Sexton et al., 2012). Telomeric and centromeric regions, as well as sex chromosomes were removed in this analysis. The RT values used in our analysis cover $85 \%$ of the human genome. The distributions of the RT values aggregated across all samples are shown in Figure 1A. RT data were normalized using the limma package (Smyth, 2005) in R

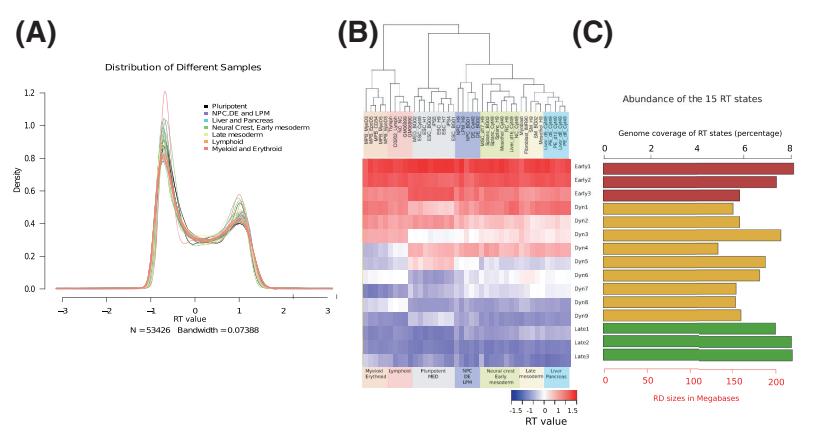

Fig. 1. Properties of RT values and overview of 15 RT states. (A) Global RT value distributions along the 42 datasets of 25 cell types. (B) RT values of the 15 RT states along the 42 datasets. The genome was decomposed into 15 RT states based on the RT program of each sample. The average value for each state is displayed in the heatmap and the scale is displayed at the bottom. The dendrogram was produced by hierarchical clustering of the samples. We saw that the RT states Early1 and Early2 replicate early across almost all cell types; Early3 shows significantly earlier RT in pluripotent cell types than in differentiated cell types. The RT states which change between cells are called dynamic RT state (Dyn). RT state Dyn1 displays significantly earlier RT in differentiated cell types. For RT state Dyn2, pluripotent cells and myeloid/erythroid and lymphoid cells show significantly earlier RT than other cell types. In RT state Dyn3, pluripotent cells show later RT than other cell types. In RT state Dyn4, myeloid/erythroid and lymphoid cells show later RT than other cell types. RT state Dyn5 shows a trend similar to the Early3 RT state, with intermediate RT in pluripotent cells but significantly later RT in all other cell types. RT Dyn6 shows late RT in pluripotent cells and intermediate RT for the rest. RT state Dyn7 shows late RT among myeloid/erythroid and lymphoid cell types and intermediate RT elsewhere. RT state Dyn8 shows the opposite pattern in Dyn8, i.e. intermediate RT among myeloid/erythroid and lymphoid cells but late RT for the rest. RT state Dyn9 shows the opposite pattern in Dyn6, i.e. intermediate RT in pluripotent cells but late RT elsewhere. RT states Late 1-3 show late RT across all cell types in general. In Late1, late mesoderm and liver/pancreas cells tend to show less late RT compared to other cell types, whereas Late2 shows less late RT in myeloid/erythroid cell types compared to other cell types. (C) Percentage and size (in mega bases, red axis) of genome coverage for each of the $15 \mathrm{RT}$ states 
and rescaled to the same range using quantile normalization (Irizarry et al., 2003).

\subsection{HMM}

Like the chromHMM model proposed by Ernst and Kellis to call chromatin states (Ernst and Kellis, 2010, 2012; Ernst et al., 2011), we use a multivariate HMM to capture the underlying structure of the 42 RT programs. Our model assumes a fixed number-15, of hidden states $K$ (the selection of $K$ is discussed in detail in a subsequent section). For each hidden state, we use a distinct normal distribution (emission probabilities) to characterize the RT values (at the bin level) from each RT program. Specifically, for each of the $K$ hidden states, there are two parameters: $\mu_{k}$ and $\sigma_{k}^{2}$, denoting the mean and variance of the normal distribution in hidden state $k_{(k=1, \ldots . K)}$ that the RT values follow. Although many of the datasets and cell types are closely related, for the sake of mathematical simplicity, following chromHMM, we treat the RT values obtained from different cell types as independent condition on the assigned RT state at the locus. For a specific chromosome $C$, The full likelihood of all of the observed RT values $x$ and together with key HMM parameters $a$ (initial probabilities), $b$ (transition probabilities) can be expressed:

$$
\begin{aligned}
P\left(x \mid a, b, \mu, \sigma^{2}\right)= & \sum_{s_{C} \in S_{C}} a_{s_{C_{1}}}\left(\prod_{t=2}^{T_{C}} b_{S_{c_{t-1}}, S_{c_{t}}}\right) \\
& \left(\prod _ { k = 1 } ^ { K } \prod _ { s _ { c _ { t } } = k } \left(\frac{1}{\sqrt{2 \pi} \sigma_{k}} e^{\left.\left.-\frac{\left(x_{\left.s_{c_{t}}-\mu_{k}\right)^{2}}^{2 \sigma_{k}^{2}}\right.}{k}\right)\right)}\right.\right.
\end{aligned}
$$

Where $N($,$) is the density function for normal distribution c_{t}$ denotes a $50 \mathrm{~kb}$ bin on chromosomes where $t=1, \ldots T c$. Let $b_{i, j}$ denotes the probability of transitioning from state $i$ to $j$ where $i_{(i=1, \ldots, K)}$ and $j_{(j=1, \ldots, K)}$. We also have parameters $a_{i_{(i=1, \ldots, K)}}$ which denote the probability that the state of the first interval on the chromosome is $i$. Let $s_{c} \in$ $S_{C}$ be an unobserved state sequence through chromosome $c$ and $S_{C}$ be the set of all possible state sequences. Let $s_{c_{t}}$ denotes the unobserved state on chromosome $c$ at location $t$ for state sequence $s_{c}$. The model has a similar structure as chromHMM method (Ernst et al., 2011) but customized for fitting RT data. We use R package depmix4 to conduct inference on the HMM (Visser, 2010). Once the HMM parameters are estimated and $50 \mathrm{~kb}$ bins along the genomes are assigned to different RT states. We group all bins belong to the same RT states together and obtain average RT values for each RT state in every cell type. We next perform a two-way clustering on these average RT values, and ordered the $15 \mathrm{RT}$ states such that the first one has the earliest RT values and the last one has the latest RT values. By doing this, the 15 RT states are ordered from early RT to late RT.

\subsection{Compare to chromatin states}

Chromatin state annotations of nine ENCODE cell types (Ernst et al., 2011) defined on nine different epigenetic marks were downloaded from the chromHMM website (http://compbio.mit.edu/ ChromHMM/). The nine cell lines are: GM12878 (B-lymphocyte), H1-hESC (hESC), HepG2 (hepatocellular carcinoma), HMEC (mammary epithelial cells), HSMM (skeletal muscle myoblasts), K562 (leukemia), NHEK (epidermal keratinocytes), NHLF (lung fibroblasts) and HUVEC (umbilical vein endothelial cells). The chromHMM 15 chromatin states are: active promoter, weak promoter, inactive/poised promoter, strong enhancer, strong enhancer, weak/poised enhancer, weak/poised enhancer, insulator, transcriptional transition, transcriptional elongation, weak transcribed, Polycomb-repressed, heterochromatin (Het), low signal, repetitive/ Copy Number Variation (CNV) 1 and repetitive/CNV 2. The 15 chromatin states were defined based on the occurrence of a combination of eight different epigenetic marks (H3K27ac, H3K27me3, H3k36me3, H3K4me1, H3K4me2, H3K4me3, H3K9ac and H3K20me1) and CTCF binding sites. To compare RT states and chromatin states, the proportion of the genomes corresponds to the 15 chromatin states and the 15 RT states were obtained with the following formula: $\log 10\left(\frac{r_{i j}}{p_{i} * q_{i}}\right)$. With chromatin state proportions: $p_{\left(p_{1}, \ldots, p_{15)}\right.}$ and RT state proportions: $q_{\left(q_{1}, \ldots, q_{15)}, r_{\left.i_{(i=1, \ldots}, 15\right)}, j_{(j=1, \ldots, 15)}\right.}$ corresponds to the combination of chromatin states $i$ and RT state $j$.

\subsection{Compare to other annotations}

In addition to chromatin states, multiple alternative annotation methods have been developed (Filion et al., 2010; Hoffman et al., 2012, 2013; Libbrecht et al., 2015, 2018; Marco et al., 2017; Zhang and Hardison, 2017). In this study, we also compared RT states with three additional types of chromatin annotations. Zhang and Hardison introduced an advanced genome segmentation and annotation method named IDEAS that decompose the genome into 20 different states (Zhang and Hardison, 2017). To compare, we use IDEAS annotation from the same nine cell types as in chromHMM analysis (file available from http://bx.psu.edu/ yuzhang/Roadmap_ ideas/). Hoffman et al. combined chromHMM (Ernst et al., 2011) and Segway (Hoffman et al., 2012) annotation to produce an integrated annotation (Hoffman et al., 2013) with seven states for six ENCODE cell lines (GM12878, H1-hESC, HepG2, HELA, K562 and HUVEC, files available on http://ftp.ebi.ac.uk/pub/databases/ ensembl/encode/integration_data_jan2011/byDataType/segmentations/ jan2011/hub). Libbrecht et al. developed GBR (Libbrecht et al., 2015) to decompose the genome into five different types of domains. GBR annotations on eight different cell lines are obtained from https://noble.gs.washington.edu/proj/gbr/. To compare with these alternative annotations, we again apply the method described above for comparing chromatin states with RT states.

\subsection{Gene features analysis}

The detection of the gene features in each $50 \mathrm{~kb}$ bin was performed on $\mathrm{Hg} 19$ General Feature Format file (file available on www.genco degenes.org). There are four different global gene features: protein coding (contains an open reading frame), non-coding RNA (ncRNA), long ncRNA (lncRNA) and pseudogenes (similar to known proteins but contain a frameshift and/or stop codon, which disrupts the open reading frame).

\subsection{Transcriptome analysis on protein -coding gene}

Expression level measured by the Reads Per Kilobase of transcript per Million mapped reads (RPKM) of RNA sequencing data of protein coding genes of 17 cell lines were downloaded from REMC (http:// www.roadmapepigenomics.org/) (Bernstein et al., 2010). The 17 cell types include the nine ENCODE cell types used in the chromHMM analysis plus additional eight cell types overlapping with the 42 cell lines used to construct the RT states: H1 BMP4 derived trophoblast, $\mathrm{H} 1$ derived mesenchymal stem, $\mathrm{H} 1$ derived neuronal progenitor, hESC derived CD184 endoderm, hESC derived CD56 ectoderm, hESC derived CD56 mesoderm and HELA. The RPKM value of the protein -coding genes that located inside regions corresponding to each of the 15 RT states were calculated, then their average expression levels in is computed in $\log$ scale as $\log 10\left(\mathrm{RPKM}_{\text {average }}\right)$.

\subsection{Gene ontology term enrichment analysis}

Gene ontology (GO) (Ashburner et al., 2000) term enrichment analysis is performed using Proteinside (Kaspric et al., 2015). 
The analysis is realized using the lists of protein -coding genes belong to each of the $15 \mathrm{RT}$ states compiled for the RNA-seq analysis. The 20 GO terms most enriched in each of the $15 \mathrm{RT}$ states were used to analyze the specificity and characteristics of genes localized in each RT state. We also computed the average RT score for all the GO terms in the Biological Process (BP) and Molecular Function (MF) categories (Ashburner et al., 2000). Since the RT states are ordered from Early to Late, the average RT score for a group of genes reflects their RT.

\subsection{Three-dimensional chromosomal organization analysis}

We use high -resolution $\mathrm{Hi}-\mathrm{C}$ data (GEO accession number: GSE63525) obtained from human lymphoblastoid cells (GM12878) produced by Rao et al. (Rao et al., 2014). We count the number of contact domains found in regions corresponding to each of the 15 RT states. The same method was used to analyze the LADs in fibroblasts. The LADs data were obtained from ENCODE (Guelen et al., 2008).

\section{Results}

\subsection{RT value distribution along the genome}

RT values from Repli-chip (Hiratani et al., 2008; Ryba et al., 2011a) are the $\log 2$ ratios of signals derived from early and late samples measured by microarrays from published studies (Rivera-Mulia et al., 2015). About $85 \%$ of the genome is covered by RT values. The dynamic range has been normalized which ranges from -2.9 to 2.9 as previously described (Marchal, 2017; Rivera-Mulia et al., 2015), and their distributions in all cell types show a similar bimodal pattern with two peaks around -1 and 1 , representing respectively the genome part replicate late and early during replication (Fig. 1A). Higher RT values $(>0.3)$ were classified as early replicating; low values $(<-0.3)$ were classified as late replicating (RiveraMulia et al., 2015). Alignment of RT values from 42 datasets along a chromosome shows the global preservation of RT during development and also some deviations among cell types (Fig. 2C). As examples, we show some regions with early RT values in hESC cells but late in other cell types (the green rectangle in Fig. 2C). The opposite pattern can be observed in the blue rectangle (Fig. 2C). Such patterns illustrate the importance of statistical modeling of RT program to identify recurrent and biologically meaningful combinatorial patterns of RT values.

\subsection{Genome decomposition into RT states}

The HMM requires the specification of the number of hidden states. As in chromatin states and other annotation methods, there is no consensus on how many RT states should we define. In this study, we chose $K=15$ to balance complexity and biological interpretation. We have tested several different numbers of states $(6,10,20$ and 30) and found the results are largely comparable (Supplementary Fig. S1). Furthermore, we found that using 15 states, we can differentiate some specific and subtle RT combinatorial patterns shown in different cell types, and additionally, we are able to clustered the cell lines according to their genome-wide RT profiles in a manner consistent with the lineage origin of each cell type (Fig. 1B) (Rivera-Mulia et al., 2015). Same as chromatin state, different versions of RT state annotations are also possible when alternative numbers of total RT states are used.

We analyzed the spatial organization of the RT states, i.e. how often does the switch (RT transition of contiguous regions) between

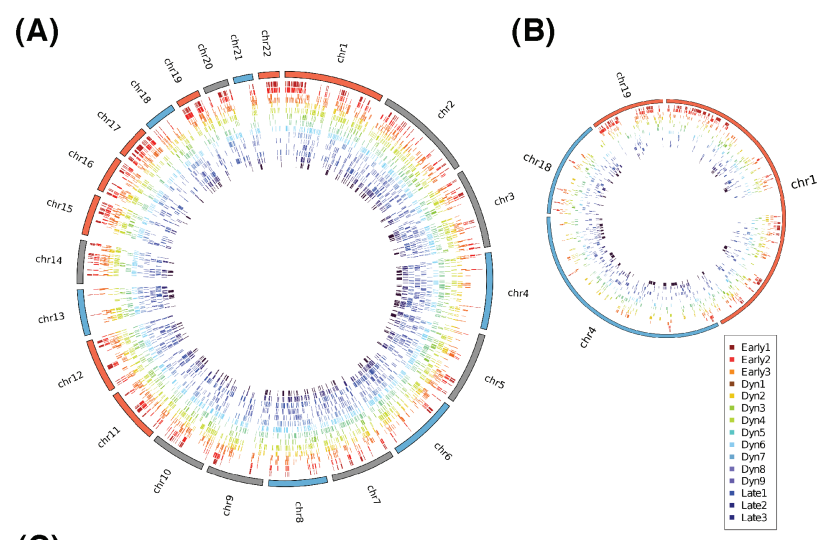

(C)

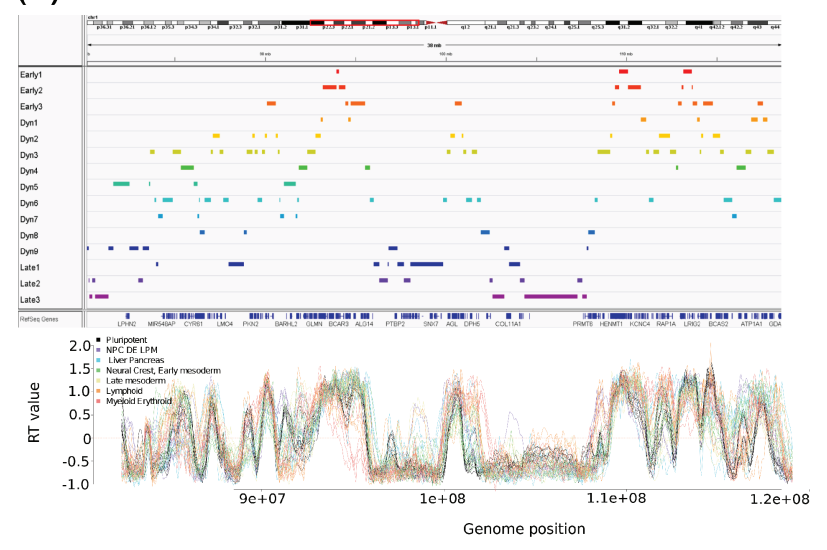

Fig. 2. Distribution of the 15 RT states along the genome. HGD chromosomes are highlighted by outside rectangles in red, LGD chromosomes are highlighted by outside rectangles in blue and all the other are in represented in gray. (A) Circos plot shows the whole genome distribution. (B) Circos plot of RT states distribution for selected HGD chromosomes (chr1 and 19) and LGD chromosomes (chr4 and 18). HGD chromosomes have larger and dense Early domains and smaller and sparse Late domains while LGD chromosomes have an opposite pattern. (C) IGV screenshot of the 15 RT states distribution and the aligned tracks in $40 \mathrm{Mb}$ segment of the chromosome 1 across all the cell lines. The $y$ axis is the RT value between -1.5 and 1.5 in this region; the $x$ axis is the genome coordinate. The red dash line shows the RT value of zero. The rectangles highlighted variations among cell lines. In the green box we observe a region with early RT value in pluripotent and MED cell lines but late or close to zero RT values in Lymphoid, Myeloid and Erythroid cell lines. In the blue box we observe a region that shows early RT values in Mesoderm, Liver and Pancreas cell lines but late RT values in pluripotent and MED cell lines

different RT states occur. By looking at the RT changes in neighboring genomic regions, we found that the RT states tend to stay unchanged for long stretches of chromosomes. It is evident from the transition matrix that the probability of staying in the same state is very high and immediately adjacent chromosomal segments are mostly classified into the same RT state (Supplementary Fig. S2A). Analysis of the average of contiguous bins for each RT states shows that the two largest domains are observed for the Early1 $(618 \mathrm{~kb})$ and Late3 $(1.16 \mathrm{Mb})$. The other RT states have value between 267 and $500 \mathrm{~kb}$ (Supplementary Fig. S2D). This is consistent with the previous findings that RDs are usually hundreds of kilobases in length (Hansen et al., 2010; Hiratani et al., 2008, 2010).

\subsection{Global RT organization of the defined states}

The RT state defined by the HMM are consistent with recent publication (Hansen et al., 2010; Hiratani et al., 2008; Rivera-Mulia 
et al., 2015) where some genome region are constitutive Early (Early 1-3) or Late (Late1-3) (Fig. 1B) in all the cell lines. Other part of the genome change during the cell differentiation (Rivera-Mulia et al., 2015), this state are also found by our results (Dyn 1-9 state). Some specific regions, also defined in Rivera-Mulia et al. (2015) change specifically between pluripotent cell line and the other, our result captures also these changes (Dyn1-6) (Fig. 1B). The distribution of the 15 RT states across the genome is shown in Figure 1C. Since we excluded centromeric regions, $\sim 85 \%$ of the genome is covered by RT state assignment. The proportion of the genome represented by each state is similar across all RT states, ranging from 132 (4.9\% genome coverage, RT state Dyn4) to $217 \mathrm{Mb}(8.1 \%$, RT state Early1), with a mean of $168 \mathrm{Mb}$.

We also analyzed the distribution of RT states based on the gene -density per chromosome (Fig. 2). The distribution reveals that high gene-density (HGD) chromosomes (chr1, 11, 12, 15, 16, 17, 19 and 22) contain regions with more early RT states and low gene-density (LGD) chromosomes (chr4, 6, 8, 13, 18, 21) contain regions with more late RT states (also see Supplementary Fig. S2B and C) (Naidoo et al., 2011). We then analyzed the base composition of the defined RT state and found a monotonically increasing trend in AT percentage from early RT states to late RT states: Early1 RT state has around $52 \%$ AT, compared to about $64 \%$ AT in the Late 3 RT state (Supplementary Fig. S2E). This is expected since most of the late RT states are located in gene-poor regions, which are marked with high AT content (Gilbert et al., 2004).

\subsection{Periodic pattern of RT states observed in part of the genome}

Illustrated by the RT states shown on a segment of chromosome 2 (Fig. 2C), we observed an interesting wave-like pattern with RT state changing from early to late, then late to early in an ordered and recurrent fashion. The patterns are very clear and pronounced in about half of the genome and are chromosome-specific (Fig. 2A and B). To study such wave-like pattern along the genome, we merged regions corresponding to the two earliest RT states (Early1 and Early2) and the two latest RT states (Late2 and Late3) into two different subsets called respectively Early domains and Late domains. We then computed for each subset (Early or Late) the distance between neighboring domains. Due to the large difference of Early and Late domains distribution between chromosomes (Supplementary Fig. S2B), we considered HGD chromosomes and LGD chromosomes separately. We found that the average size of Late domain in HGD chromosomes is $643 \mathrm{~kb}$ and the average distance between two neighboring domains is $4.27 \mathrm{Mb}$. For LGD chromosomes, the average size of Late domain is $795 \mathrm{~kb}$ and $3.83 \mathrm{Mb}$ for the average distance between two adjacent domains. For the Early domains in HGD chromosomes we found an average size of $894 \mathrm{~kb}$ and an average distance of $3.28 \mathrm{Mb}$ between two neighboring domains. In LGD chromosomes, the average size is $638 \mathrm{~kb}$ and the average distance between two adjacent ones is $5.88 \mathrm{Mb}$ (results for each chromosomes presented in Supplementary Tables S2 and S3). The analysis of the Early domain shows a significant difference between HGD and LGD in their sizes ( $P$-value $=$ 6.02E-04) and distances $(P$-value $=1.01 \mathrm{E}-02)$. But neither size nor distance in the Late domains showed significant differences (Supplementary Table S4).

RT states clearly illustrate that replication is consistently executed in an orderly fashion. And the RT profiles show distinct pattern between HGD and LGD chromosomes. Perhaps the HGD chromosomes replicated faster than the LGD chromosomes since the latter ones are less accessible. The imbalance of the Early and
Late domains on the HGD and LGD chromosomes are similar to that of the $\mathrm{A}$ and $\mathrm{B}$ compartments discovered on the Hi-C experimental data (Lieberman-Aiden et al., 2009; Rao et al., 2014; Sexton et al., 2012). We speculate that the distinctive and elegant periodic pattern we observed in RT indicates the chromosomal properties are highly regulated and the pattern may contribute to its ability to be readily folded in 3D space in an ordered fashion (Gibcus et al., 2018).

\subsection{Annotation features of distinct RT states}

To characterize the different RT states identified by our HMM method, we compared them to the previously described chromatin states annotated based on epigenetic modifications derived from ChIP-seq data (Ernst et al., 2011). Additionally, we also analyzed the gene expression activity inside different RT states.

First, we investigated the abundance of the four types of gene families (protein -coding, ncRNA, lncRNA and pseudogenes) in each of the 15 RT states. The results are summarized in Figure 3A and Supplementary Figure S3. We saw that the proportion of bins with annotation is high in Early states and decreased steadily when moving from Early states to Late states. Only about $50 \%$ of the Late state bins possess at least one gene annotation (of four types), down from $98 \%$ in Early states (Fig. 3A). In all RT states, protein-coding genes are the most frequently detected gene annotations except for the Late3 RT state where around $40 \%$ of the gene annotations are lncRNAs and only $35 \%$ are protein-coding genes. We found protein-coding gene annotation frequency decreased steadily across RT states from Early $(60 \%)$ to Late $(35 \%)$ (Supplementary Fig. $\mathrm{S} 3 \mathrm{~A})$. Frequency of ncRNA annotations is stable throughout all the states (Supplementary Fig. S3B) and lncRNA frequency increased steadily from Early1 $(\approx 15 \%)$ to Late3 $(\approx 35 \%$ ) (Supplementary Fig. S3C). Pseudogenes annotation is similar in Early and Dyn1-7 states and higher in the other states (Supplementary Fig. S3D). From the above, we concluded that early RT is positive correlated with the density of protein-coding genes, negatively correlated with the density of lncRNAs, but not correlated with the density of ncRNAs and pseudogenes.
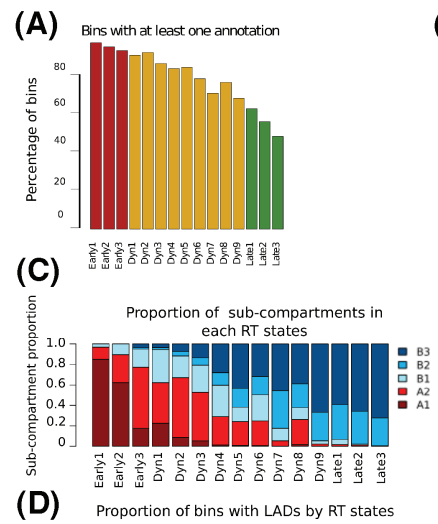

(D) Proportion of bins with LADs by RT states

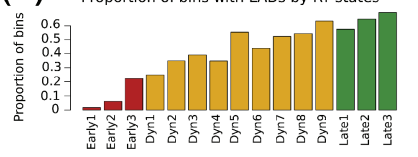

(B) Chromatin state proportion
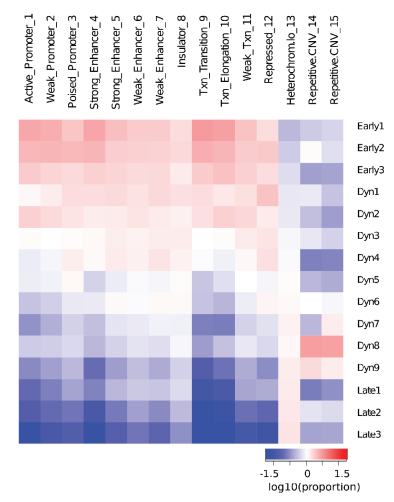

Fig. 3. Annotation features and characteristics of the different RT states. (A) Percentage of bins with at least one of the following annotation: protein-coding, ncRNA, IncRNA or pseudogene. (B) Average proportions of the Chromatin state/RT state overlap of nine cell lines. (C) Composition of the five sub-compartments in each of the 15 RT states. (D) Proportions of RT bins possessing LADs for each RT state 


\subsection{Specific chromatin architecture is detected by combining RT states and chromatin states}

It is of interest to compare the relationship between previously defined chromatin states (Ernst et al., 2011) and newly defined RT states. Since chromatin states are cell type -specific, we hope to show how the dynamic DNA chromatin organization changes with respect to the DNA replication profiles in different cell lines. Figure $3 \mathrm{~B}$ shows the average proportion of the genome belonging to the 225 different combinations of the 15 chromatin states (Ernst et al., 2011) and the 15 RT states defined in this work. Overall, we saw that except for chromatin states $13-15$, the proportions of all the other 12 chromatin states decrease steadily in regions corresponding to RT states moving from Early to Late. For chromatin state 13 (Het), the pattern is reversed as expected: the proportions keep increasing with RT states moving from Early to Late. Interestingly, we found chromatin states 14 and 15 (repetitive/CNV1 and 2) are significantly enriched (chromatin state $14: 35 \%$ and chromatin state 15: $34 \%$ ) in regions corresponding to RT state Dyn8. We also observed that these two CNV states have high proportions in Dyn9 and Dyn7. The CNVs have been shown to be correlated with later RT value and located in AT rich region (Koren et al., 2012). These are consistent with the characteristics of these three dynamic RT states which have an AT content around 60\% (Supplementary Fig. S2E) and relatively late RT. With the combination the RT states and the chromHMM states (Supplementary Fig. S4), we observed that leukemia cell line $\mathrm{K} 562$ possesses a unique pattern with relatively higher proportion of CNVs chromatin state at the early RT states than the other cell lines. Again, we saw that the Dyn7, 8 and 9 RT states are enriched in CNV states in all cell lines (Supplementary Fig. S4). In the RPKM analysis, we also observed decreased RPKM values in Dyn3 RT state in the H1 cells in comparison of the cell lines used in the chromHMM analysis (Fig. 3B). These two examples show the dynamics among RT, chromatin organization and gene expression in different cell types.

The above observation suggests that the chromatin states and RT states contain complementary information. Either one of them alone is not enough to capture all the diversities observed in different epigenomes. As an example, regions annotated by the same chromatin state may show dramatic different RT state annotation and vice versa. Thus adding RT states on top of chromatin states enable a better annotation of the genome.

\subsection{Comparing to other chromatin annotations}

We also compare RT states with three additional chromatin annotations (Hoffman et al., 2013; Libbrecht et al., 2015; Zhang and Hardison, 2017) using the same method as above. Heatmaps showing enrichment/depletion between RT states and each of the three additional annotations are shown in Supplementary Figure S5. We found that most of the functional IDEAS states (Zhang and Hardison, 2017) show enrichment in the early RT states, and depletion in late RT states except for quiescent (Quiet), Het and Het weak state regions. Interestingly, the only RT state that shows enrichment for Het IDEAS state is Dyn8. Additionally, ZNF genes and repeats (ZNF_Rpts) show the most enrichment in RT states Dyn2 and Dyn5. For Hoffman et al.'s method (Hoffman et al., 2013), we found all except the Repressive (R) state show enrichment in early RT states and depletion in late RT state. We noticed that Dyn2 and Dyn3 RT states each show almost complimentary enrichment pattern: each RT state is enriched in three different states, but the six states have no overlap. For GBR (Libbrecht et al., 2015), we found Quiet domains (QUI_1) and constitutive Het domains (CON_2) states are mostly enriched in late RT states, facultative Het domains (FAC_3) states are enriched in Dynamic RT states and broad expression domains (BRD_4) and specific expression domains (SPC_5) states are mostly enriched in early RT states.

\subsection{Gene expression and GO term enrichment analysis in the RT states}

For each cell line, we computed the average gene expression (in RPKM) for each RT state (Supplementary Fig. S6). We first observed that the cell lines of the same type tend to cluster together. As expected, protein-coding genes located inside regions belonging to the three Early states are highly expressed. Genes located in genomic regions belonging to the Late3 RT state have the lowest expression values in all cell lines tested. Dynamic states are more complex and variable, with intermediate expression values between Early and Late states. These observations confirmed that regions with higher RT values tend to have higher gene expression levels.

To find out if there is any specific pathway or gene set that is enriched in different RT domains, we conducted a GO term enrichment analysis on the genes overlapping with each of the $15 \mathrm{RT}$ states. The 20 most significantly enriched GO terms for each RT state are presented in Supplementary Table S5. We also calculated the average RT state score (since RT state number is ordinal) for each GO term and report result in Supplementary Table S5. We observed that Early states are enriched in processes involved in housekeeping pathways, like cell division, apoptosis and transcription regulation (Tables 1 and 2 and Supplementary Table S5). That is consistent with the RT score analysis which showed lower values for the same pathways [apoptotic process (3.99), protein phosphorylation (3.74), cell division (3.7)] (Supplementary Table S5). Late RT states are enriched with GO terms about stress response or defense response, and are enriched in processes involved in neural cell regulation, and tissue development. These pathways possess high average RT scores (>9) (Supplementary Table S5). Dyn RT states are enriched in transcription regulation. Finally, we also observed heart, brain, epidermal growth factor development and nervous system development GO terms enriched for Dyn RT states, which is consistent with the fact that the Dyn RT states are the RT states which change between different cell types.

\subsection{Representation of sub-compartment domains and LAD in the RT states}

Genome-wide chromatin conformation capture (Hi-C) experiments (Lieberman-Aiden et al., 2009) have revealed TADs (Dixon et al., 2012; Hou et al., 2012; Sexton et al., 2012). The majority of these domains were conserved across cell types, but the inter-TAD interactions are found to be cell type-specific (Dixon et al., 2012; Lieberman-Aiden et al., 2009; Nora et al., 2012). Comparing TAD and RT states allows us to analyze changes in the 3D chromatin organization that occur during cell differentiation which respect to RT states. Furthermore in higher organization of the genome was identified, A and B compartments characterized by the spatial segregation of open and closed chromatin (Lieberman-Aiden et al., 2009).

Rao et al. analyzed the A and B compartments and detected six sub-compartments based on their contact, architecture and epigenetics features: A1 and A2 (part of the A compartment) and B1, B2, B3 and $\mathrm{B} 4$ (part of the B compartment). In this analysis, we ignored the B4 compartment, which is defined only for by a handful of regions, containing many KRAB-ZNF super-family genes and $0.01 \%$ of the bins of each RT possess overlapped with this sub-compartment (Rao et al., 2014). The A1 sub-compartment is characterized by a high 
Table 1. RT state analysis on BP GO terms

\begin{tabular}{lllll} 
GO term & ID & Number of genes & Avg RT score & Stdev RT score \\
\hline Translational initiation & GO: 0006413 & 123 & 2.683 & 2.188 \\
Regulation of mRNA stability & GO: 0043488 & 106 & 2.953 & 2.424 \\
rRNA processing & GO: 0006364 & 188 & 2.979 & 2.379 \\
mRNA splicing, via spliceosome & GO: 0000398 & 215 & 3.005 & 2.695 \\
Tumor necrosis factor-mediated signaling pathway & GO: 0033209 & 118 & 3.186 & 2.749 \\
Regulation of complement activation & GO: 0030449 & 104 & 8.260 & 3.739 \\
Complement activation, classical pathway & GO: 0006958 & 137 & 9.139 & 3.204 \\
Detection of chemical stimulus involved in sensory perception of smell & GO: 0050911 & 391 & 9.486 & 3.664 \\
Sensory perception of smell & GO: 0007608 & 226 & 9.535 & 4.019
\end{tabular}

Note: Light (top portion, green in the online version) and dark (bottom portion, red in the online version) background colors of the table represent respectively the GO term enriched in Early or Late state. The table highlights the most significant results (additional results can be found in the Supplementary Table S5).

Table 2. RT state analysis MF GO terms

\begin{tabular}{lllll} 
GO term & ID & Number of genes & Avg RT score & Stdev RT score \\
\hline Structural constituent of ribosome & GO: 0003735 & 159 & 3.157 & 2.608 \\
Protein $N$-terminus binding & GO: 0047485 & 100 & 3.270 & 2.996 \\
Unfolded protein binding & GO: 0051082 & 115 & 3.313 & 2.645 \\
RNA binding & GO: 0003723 & 1267 & 3.317 & 2.865 \\
mRNA binding & GO: 0003729 & 112 & 3.321 & 3.062 \\
G-protein coupled receptor activity & GO: 0004930 & 638 & 7.937 & 4.163 \\
Olfactory receptor activity & GO: 0004984 & 391 & 9.486 & 3.664 \\
Immunoglobulin receptor binding & GO: 0034987 & 72 & 9.500 & 3.014 \\
Antigen binding & GO: 0003823 & 158 & 9.513 & 2.987
\end{tabular}

Note: Light (top portion, green in the online version) and dark (bottom portion, red in the online version) background colors of the table represent respectively the GO term enriched in Early or Late state. The table highlights the better results (global results are The table highlights the most significant results (additional results can be found in the Supplementary Table S5).

density of actively expressed genes, and enriched with permissive histone marks (H3K36me3, H3K79me2, H3K27ac, and H3K4me1 and $\mathrm{H} 3 \mathrm{~K} 4 \mathrm{me} 3$ ) are associated with active transcription (Rao et al., 2014). We found the A1 sub-compartment highly enriched in Early1 and Early2 RT states ( 85 and $62 \%$ of bins in these two states belong to this sub-compartment respectively) and the proportion reduced steadily to almost zero for the B2 and B3 sub-comportments. In addition of the afore mentioned euchromatin marks, the A2 subcompartment is also associated with the $\mathrm{R}$ mark $\mathrm{H} 3 \mathrm{~K} 9 \mathrm{me} 3$, and is enriched in the states Early3 (59\% of the bins) and Dyn1 to Dyn4 (Dyn1: 39\%, Dyn2: 56\%, Dyn3: 45\%) (Fig. 3C) and depleted in all others. These five RT states are mainly dynamics and the genes located within these RT states are differentially expressed among different the cell lines analyzed (Supplementary Fig. S6), so it is expected to have these different states potentially associated with $\mathrm{R}$ chromatin marks. The RT states enriched at A1 and A2 subcompartments are also depleted in LADs (Fig. 3C and D), which is consistent with the fact that LADs are made of facultative Het with late replicating properties and correlated positively with $\mathrm{H} 3 \mathrm{~K} 27 \mathrm{me} 3$ and negatively with $\mathrm{H} 3 \mathrm{~K} 36 \mathrm{me} 3$. In contrast, sub-compartment B1 with characteristics of facultative Het [is found to be enriched in Dyn1 to Dyn6 RT states (Dyn1: 31\%, Dyn2: 20\%, Dyn3: 23\%, Dyn4: 30\%, Dyn5: 14\%and Dyn6: 25\%)] (Fig. 3C). The subcompartments B2 and B3, which correspond to regions that are not replicated until the end of $S$ phase, show the opposite pattern of subcompartment A1, which is enriched in late RT states including Dyn9 (27 and 64\%, respectively), Late1 to Late3 (Late1: 34 and 59\%,
Late2: 32 and 65\% and Late3: 27 and 71\%, respectively) (Rao et al., 2014) and depleted in early RT states (Fig. 3C).

LADs are formed by the interaction between nuclear lamina and the genome and are involved in genome organization. Most of the LADs are located in gene poor, $\mathrm{A} / \mathrm{T}$ rich regions, and regions enriched in repetitive elements such as SINEs and LINEs (Meuleman et al., 2013). The RT states enriched in B2, and B3 subcompartments are also enriched in LADs. As an example, around $60 \%$ of bins in Late RT states (Dyn9, Late1-3) contain LADs. In contrast, only $\sim 2.5 \%$ of bins in Early1 RT state overlap with LADs (Fig. 3D). Therefore, the Late RT states possess all the characteristics of condensed Het, late replication and dense in LAD domains. Some of the LADs are cell type -specific and defined as facultative LADs (fLADs), and others are conserved across different cell types and are referred to as constitutive LADs (cLADs) (Meuleman et al., 2013). We speculate the subset of LADs with Late RT states (Dyn9, Late1-3) that are likely to be cLADs, and are enriched in B3 subcompartment (Fig. 3C and D) and $\mathrm{A} / \mathrm{T}$ rich, gene -poor regions (Supplementary Figs S2 and S3). The fLADs, which are cell specific, on the other hand, could be localized more in the Dyn RT states, and enriched in B1 and B2 sub-compartment, which are correlated with the facultative Het.

\section{Discussion}

Genome-wide datasets constitute a valuable tool for genome annotation of distinct chromatin functional properties, especially the 
non-coding part of the genome. Histone modification, DNA methylation, chromatin accessibility and disease association have all been used to provide functional annotation (Boyle et al., 2012; Chen and Qin, 2016; Dunham et al., 2012; Fu et al., 2014; Ward and Kellis, 2012, 2016). Since RT is a fundamental BP, closely related to chromatin organization and transcription regulation dynamics during cell fate commitment, we believe RT can supplement the current functional annotation of the genome. Our RT state concept is inspired by the chromHMM work of Ernst and Kellis (Ernst and Kellis, 2010, 2012; Ernst et al., 2011). They define chromatin states by applying HMM on ChIP-seq data of histone modifications, together with histone variants (H2A.Z), Polymerase II and CTCF. The chromatin state defined possesses specific functional, experimental, conservation, annotation and sequence-motif enrichments.

In this work, we develop a novel HMM to classify genome-wide RT programs from 42 datasets into $15 \mathrm{RT}$ states such that these RT states can be used to annotate the genome. The RT state annotation improves over the RD annotation (constitutive and developmental) introduced earlier (Dileep et al., 2015; Rivera-Mulia et al., 2015) by offering richer and more quantitative annotation with better resolution. Although both the RT and the chromatin states are developed from applying HMM to demarcate the chromosomes into segments of diverse flavors, the RT and chromatin states have different properties and are complementary to each other. In addition to the source of information, the major differences between RT and chromatin states are 4-fold: (1) while chromatin states change from one cell type to another, RT states is not cell type -specific. This is because RT states are summarized from a compendium of cell types and developmental stages. (ii) the chromatin states are categorical whereas the RT values are ordinal and quantitative, smaller RT state values indicates earlier replication; (iii) chromatin state is fine grain type annotation, the resolution is typically $200 \mathrm{bp}$. RT states are defined on a much larger scale, annotated at $50 \mathrm{~kb}$ level throughout the genome as RT status typically remain stable over a long stretch of the chromosome in most cell types; (iv) the abundance of the 15 chromatin states vary dramatically while the 15 RT states have similar proportions across the genome.

An interesting question is why we bother to call on sophisticated statistical modeling to obtain the $15 \mathrm{RT}$ states. Whether similar results can be obtained by simply averaging the RT signals across all cell types we have. By definition, for identification purpose, the 15 RT states are ordered from early to late in terms of their average RT values across the genome and cell types. Hence as expected, some of the results, like the gradual pattern of shifting in RT for the 15 RT states, can be conceivably produced by averaging RT values. But importantly, the functional annotation offered by the RT states, which is the main motivation for developing it, cannot be produced by averaging RT values. This is because RT is cell type-specific, a genomic locus may be replicated much earlier in a specific cell type than others. Two loci in the genome may have the same average RT values across all cell types, but their RT pattern may be completely different for any given cell type. RT states are designed to distinguish such cell type-specific RT patterns even when the average RT values are the same. For example, in Figure $1 \mathrm{~B}$ which shows the average RT values in each all cell types across the $15 \mathrm{RT}$ states, Dyn8 RT state corresponds to neutral RT in Myeloid and Lymphoid cells and late RT in Pluripotent cells. On the other hand, adjacent Dyn9 RT state shows the complete opposite pattern for these cell types. Since the RT pattern in the remainder cell types is quite similar, we will not be able to distinguish the two RT states if we use the average RT values across all cell types (Supplementary Fig. S7).
The RT states defined by the HMM method illustrate functional properties of the genome. Early RT states possessed open chromatin features with HGD, enriched in A1 and A2 sub-compartments, enriched in epigenetic marks associated with open chromatin (H3K36me3, H3K79me2, H3K27ac, H3K4me1) and depleted in LADs. Late RT states possessed Het properties, rich in pseudogenes and lncRNAs, weak in genes and enriched in LADs and B2 and B3 sub-compartments. Dynamic RT states include chromosomal regions that change RT during development (Liu et al., 2016; Rivera-Mulia et al., 2015) and were linked to more variable gene expression activity, chromatin organization, GO terms relative to the development of tissues and possess the characteristics of facultative Het. The novel RT state concept we propose here has many applications. For example, an application could be to calculate the average RT scores for a set of genomic entities such as genes belong to a particular gene set of pathway. This is possible because the RT states are ordinal (low RT state number indicates early RT and high RT state number indicates late RT). An unusually low or high average RT scores suggest RT connection among these entities. We can also compare the RT scores between two groups of genomic entities of interest to see if they share the same RT profile.

In addition to the reductionist style summary of multiple series of RT values from different cell types, RT states also offer easy visualization of RT data as shown in the wave-like pattern observed in Figure 2C, suggesting early or late replicating genomic regions are arranged in an ordered fashion. Comparing to plots of individual RT values shown at the bottom of the Figure $2 \mathrm{C}$, the pattern of RT states is rather clean and smoother, and a single track of RT states is sufficient to give researchers an overview of the local RT landscape as oppose to 42 , highlighting the benefit of the model-based approach we took to obtain RT states. As shown in Figure 3C, the RT states are closely related to the $\mathrm{A} / \mathrm{B}$ compartment concept derived from $\mathrm{Hi}-\mathrm{C}$ data, early RT states match compartment A while late states correspond to compartment $\mathrm{B}$. This also corroborate well with the computational derived 3D chromosomal organization constructed based on Hi-C data [Fig. 3F in Hu et al. (2013a)], confirming the intimate relationship among RT, A/B compartments in the chromosomes and $3 \mathrm{D}$ shapes of the chromosomes (Ryba et al., 2010).

Various computational methods have been proposed to identify RDs using RT profile data (Hansen et al., 2010; Pope et al., 2014a; Ryba et al., 2010). In a recent study, Liu et al. described an advanced deep neural network (DNN)-based method named DNNHMM that is capable of identifying multiple RDs genome-wide (Liu et al., 2016). Using manually labeled training data, Liu et al. apply DNN on Repli-seq data to assign four major types (and 14 subtypes) of RDs throughout the genome. On top of the methodology, the biggest difference between our method and DNN-HMM (and other existing RD calling methods) is that DNN-HMM is designed to call $\mathrm{RDs}$ in a single cell type using replication profiling data from that cell type whereas we are trying to define and call RT states across a compendium of cell types, emphasizing the homogeneity and heterogeneity of the RT programs among cells from different developmental lineages and stages. Each of the 15 RT states represents a particular combination of RDs from multiple cell types. As a result, the $15 \mathrm{RT}$ states can be used to annotate the diverse properties of the genome holistically, not just for a single cell type. We believe our method represents the first attempt to jointly model multiple RT profiling data in order to reveal underlying biological insights.

Many studies have been conducted using epigenetics to annotate the genome (Chan et al., 2017; Ernst et al., 2011; Hoffman et al., 2012, 2013; Libbrecht et al., 2015, 2018; Marco et al., 2017; Zhang and Hardison, 2017), especially the non-coding part, RT states can 
add a new dimension to the annotation, and can elucidate the changes in organization during differentiation and development. This work could also shed light on the dynamics of the 3D chromatin organization during differentiation by correlating the RT states and the Hi-C data. Data with better resolution (smaller size of fragments) of the RT data will allow for better definition of the domains of replication and refinement of our models. This could permit future analysis of the RT of specific promoters involved in cell differentiation.

\section{Acknowledgements}

We thank two anonymous reviewers for their constructive comments. We thank the members of the Gilbert Lab and the Corces Lab for sharing the RT data and for discussions. We thank Professor Christophe Tatout in the GReD institute, and Michael Holden Nichols in the Corces lab and Philip A. Guerrero for comments on the draft of the manuscript.

\section{Funding}

This work was supported by National Institutes of Health [P01 GM085354].

Conflict of Interest: none declared.

\section{References}

Ashburner,M. et al. (2000) Gene ontology: tool for the unification of biology. The Gene Ontology Consortium. Nat. Genet., 25, 25-29.

Bae,J.B. (2013) Perspectives of international human epigenome consortium. Genomics Inform., 11, 7-14.

Bernstein,B.E. et al. (2010) The NIH Roadmap Epigenomics Mapping Consortium. Nat. Biotechnol., 28, 1045-1048.

Boyle,A.P. et al. (2012) Annotation of functional variation in personal genomes using RegulomeDB. Genome Res., 22, 1790-1797.

Chan,R.C.W. et al. (2017) Segway 2.0: Gaussian mixture models and minibatch training. Bioinformatics, 34, 669-671.

Chen,L. and Qin,Z.S. (2016) traseR: an R package for performing trait-associated SNP enrichment analysis in genomic intervals. Bioinformatics, 32, 1214-1216.

Dileep,V. et al. (2015) Large-scale chromatin structure-function relationships during the cell cycle and development: insights from replication timing. Cold Spring Harb. Symp. Quant. Biol., 80, 53-63.

Dixon,J.R. et al. (2012) Topological domains in mammalian genomes identified by analysis of chromatin interactions. Nature, 485, 376-380.

Dunham,I. et al. (2012) An integrated encyclopedia of DNA elements in the human genome. Nature, 489, 57-74.

Ernst,J. and Kellis,M. (2010) Discovery and characterization of chromatin states for systematic annotation of the human genome. Nat. Biotechnol., $28,817-825$.

Ernst,J. and Kellis,M. (2012) ChromHMM: automating chromatin-state discovery and characterization. Nat. Methods, 9, 215-216.

Ernst,J. et al. (2011) Mapping and analysis of chromatin state dynamics in nine human cell types. Nature, 473, 43-49.

Filion, G.J. et al. (2010) Systematic protein location mapping reveals five principal chromatin types in Drosophila cells. Cell, 143, 212-224.

Fu,Y. et al. (2014) FunSeq2: a framework for prioritizing noncoding regulatory variants in cancer. Genome Biol., 15, 480.

Gerhardt,J. et al. (2014) Cis-acting DNA sequence at a replication origin promotes repeat expansion to fragile X full mutation. J. Cell Biol., 206, 599-607.

Gibcus,J.H. et al. (2018) A pathway for mitotic chromosome formation. Science, 359, eaao6135.

Gilbert,N. et al. (2004) Chromatin architecture of the human genome: gene-rich domains are enriched in open chromatin fibers. Cell, 118, 555-566.

Guelen,L. et al. (2008) Domain organization of human chromosomes revealed by mapping of nuclear lamina interactions. Nature, 453, 948-951.
Hansen,R.S. et al. (2010) Sequencing newly replicated DNA reveals widespread plasticity in human replication timing. Proc. Natl. Acad. Sci. U S A, 107, 139-144.

Hatton,K.S. et al. (1988) Replication program of active and inactive multigene families in mammalian cells. Mol. Cell Biol., 8, 2149-2158.

Hiratani,I. et al. (2010) Genome-wide dynamics of replication timing revealed by in vitro models of mouse embryogenesis. Genome Res., 20, 155-169.

Hiratani,I. et al. (2008) Global reorganization of replication domains during embryonic stem cell differentiation. PLoS Biol., 6, 2220-2236.

Hiratani,I. et al. (2009) Replication timing and transcriptional control: beyond cause and effect-part II. Curr. Opin. Genet. Dev., 19, 142-149.

Hoffman,M.M. et al. (2012) Unsupervised pattern discovery in human chromatin structure through genomic segmentation. Nat. Methods, 9, 473-476.

Hoffman,M.M. et al. (2013) Integrative annotation of chromatin elements from ENCODE data. Nucleic Acids Res., 41, 827-841.

Hou,C.H. et al. (2012) Gene density, transcription, and insulators contribute to the partition of the Drosophila genome into physical domains. Mol. Cell, $48,471-484$.

Hu,M. et al. (2013a) Bayesian inference of spatial organizations of chromosomes. PLoS Comput. Biol., 9, e1002893.

Hu,M. et al. (2013b) Understanding spatial organizations of chromosomes via statistical analysis of Hi-C data. Quant. Biol., 1, 156-174.

Irizarry,R.A. et al. (2003) Exploration, normalization, and summaries of high density oligonucleotide array probe level data. Biostatistics, 4, 249-264.

Kaspric,N. et al. (2015) Protein Function Easily Investigated by Genomics Data Mining Using the ProteINSIDE Online Tool. Genomics Comput. Biol., 1, e16.

Koren,A. et al. (2012) Differential relationship of DNA replication timing to different forms of human mutation and variation. Am. J. Hum. Genet., 91, 1033-1040.

Libbrecht,M.W. et al. (2015) Joint annotation of chromatin state and chromatin conformation reveals relationships among domain types and identifies domains of cell-type-specific expression. Genome Res., 25, 544-557.

Libbrecht,M.W. et al. (2018) A unified encyclopedia of human functional DNA elements through fully automated annotation of 164 human cell types. bioRxiv.

Lieberman-Aiden,E. et al. (2009) Comprehensive mapping of long-range interactions reveals folding principles of the human genome. Science, 326 , 289-293.

Liu,F. et al. (2016) De novo identification of replication-timing domains in the human genome by deep learning. Bioinformatics, 32, 641-649.

Marchal,C.S. et al. (2017) Repli-seq: genome-wide analysis of replication timing by next-generation sequencing. bioRxiv.

Marco,E. et al. (2017) Multi-scale chromatin state annotation using a hierarchical hidden Markov model. Nat. Commun., 8, 15011.

Meuleman,W. et al. (2013) Constitutive nuclear lamina-genome interactions are highly conserved and associated with A/T-rich sequence. Genome Res., 23, 270-280.

Naidoo,N. et al. (2011) Human genetics and genomics a decade after the release of the draft sequence of the human genome. Hum. Genomics, 5, $577-622$.

Nora,E.P. et al. (2012) Spatial partitioning of the regulatory landscape of the $\mathrm{X}$-inactivation centre. Nature, 485, 381-385.

Pope,B.D. and Gilbert,D.M. (2013) The replication domain model: regulating replicon firing in the context of large-scale chromosome architecture. $J$. Mol. Biol., 425, 4690-4695.

Pope,B.D. et al. (2014) Topologically associating domains are stable units of replication-timing regulation. Nature, 515, 402-405.

Pope,B.D. et al. (2011) DNA replication timing is maintained genome-wide in primary human myoblasts independent of $\mathrm{D} 4 \mathrm{Z} 4$ contraction in FSH muscular dystrophy. PLoS One, 6, e27413.

Rao,S.S.P. et al. (2014) A 3D map of the human genome at kilobase resolution reveals principles of chromatin looping. Cell, 159, 1665-1680.

Rivera-Mulia,J.C. et al. (2015) Dynamic changes in replication timing and gene expression during lineage specification of human pluripotent stem cells. Genome Res., 25, 1091-1103. 
Rivera-Mulia,J.C. et al. (2017) DNA replication timing alterations identify common markers between distinct progeroid diseases. Proc. Natl. Acad. Sci. U S A, 114, E10972-E10980.

Rivera-Mulia,J.C. and Gilbert,D.M. (2016) Replicating large genomes: Divide and Conquer. Mol. Cell, 62, 756-765.

Ryba,T. et al. (2012) Abnormal developmental control of replication-timing domains in pediatric acute lymphoblastic leukemia. Genome Res., 22, 1833-1844.

Ryba,T. et al. (2010) Evolutionarily conserved replication timing profiles predict long-range chromatin interactions and distinguish closely related cell types. Genome Res., 20, 761-770.

Ryba,T. et al. (2011a) Genome-scale analysis of replication timing: from bench to bioinformatics. Nat. Protoc., 6, 870-895.

Ryba,T. et al. (2011b) Replication timing: a fingerprint for cell identity and pluripotency. PLoS Comput. Biol., 7, e1002225.

Sasaki,T. et al. (2017) Stability of patient-specific features of altered DNA replication timing in xenografts of primary human acute lymphoblastic leukemia. Exp. Hematol., 51, 71-82 e73.
Sexton, T. et al. (2012) Three-dimensional folding and functional organization principles of the Drosophila genome. Cell, 148, 458-472.

Smyth,G.K. (2005) limma: linear models for microarray data. In: Gentleman,R. et al. (eds) Bioinformatics and Computational Biology Solutions Using $R$ and Bioconductor. Statistics for Biology and Health. Springer, New York, NY, pp. 397-420.

Visser,I.S.M. (2010) depmixS4: an R Package for Hidden Markov Models. J. Stat. Softw., 36, 1-21.

Ward,L.D. and Kellis,M. (2012) HaploReg: a resource for exploring chromatin states, conservation, and regulatory motif alterations within sets of genetically linked variants. Nucleic Acids Res., 40, D930-D934.

Ward,L.D. and Kellis,M. (2016) HaploReg v4: systematic mining of putative causal variants, cell types, regulators and target genes for human complex traits and disease. Nucleic Acids Res., 44, D877-D881.

Zhang,Y. and Hardison,R.C. (2017) Accurate and reproducible functional maps in 127 human cell types via 2D genome segmentation. Nucleic Acids Res., 45, 9823-9836. 